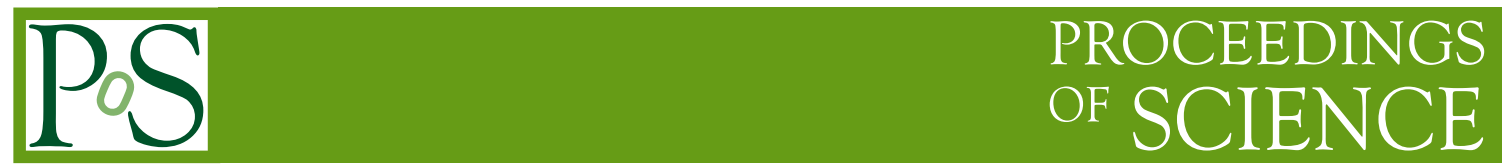

\title{
Overview of LHC results
}

\section{Günther Dissertori*}

Institute for Particle Physics, ETH Zurich, Switzerland

E-mail: dissertoriephys.ethz.ch

This review summarizes some of the most relevant recent results obtained by the LHC experiments. Emphasis is put on tests of the Standard Model, on heavy flavour and top quark physics, on searches for physics beyond the Standard Model and finally on the search for the Higgs boson.

Loops and Legs in Quantum Field Theory, 11th DESY Workshop on Elementary Particle Physics April 15-20, 2012

Wernigerode, Germany

\footnotetext{
* Speaker.
} 


\section{Introduction}

The recent months have been characterized by a most impressive amount of results presented by the LHC experiments. Most of these new measurements are based on the statistics collected during the 2011 LHC run. Here an overview of some of the highlights will be given, with focus on hard scattering processes, searches for new physics and searches for the Higgs boson. The plethora of results on soft, forward and diffractive physics, as well as on heavy ion collisions, can unfortunately not be covered here. A recent, more comprehensive, review can, e.g., be found in Ref. [1].

None of the results presented below would have been possible without the excellent performance of our tools, namely the accelerator and detectors. In 2011, the LHC has achieved several important milestones and even world records, mostly in terms of beam intensities, instantaneous and integrated luminosities, both for the p-p and the heavy ion (HI) running. Overall, during the proton run the LHC has delivered about $12.5 \mathrm{fb}^{-1}$ to its experiments, with the largest fraction (about $5.5 \mathrm{fb}^{-1}$ each) to the two general purpose detectors ATLAS and CMS, and smaller amounts, because of luminosity leveling, to LHCb $\left(1.2 \mathrm{fb}^{-1}\right)$ and ALICE $\left(0.005 \mathrm{fb}^{-1}\right)$. This became possible thanks to an increase by a factor of 20 in the p-p peak luminosity, compared to the 2010 run.

\section{Tests of perturbative QCD}

Measurements of hard-scattering cross sections, with jets, photons or vector bosons in the final state, are interesting because of several reasons: (i) it allows probing higher-order predictions of perturbative QCD for the hard-scattering part of the overall process; (ii) parton distribution functions can be constrained; (iii) Standard Model (SM) predictions can be tested, in particular QCD calculations, as implemented in various codes and Monte Carlo (MC) generators, for processes which are important backgrounds for new physics searches. For a more extensive review of this subject we refer to, e.g. [2].

A central component of those measurements, which contain jets in the final state, is the excellent control of the systematic uncertainty due to the jet energy scale. This is essential because of the nature of the steeply falling cross sections as a function of the jet $p_{T}$. By now the LHC experiments master this effect already at a remarkable level of precision [3, 4], e.g., around $2 \%$ or even better for central jets and a $p_{T}$ range of about 50 to several hundred $\mathrm{GeV}$.

Concerning jet production at the LHC, new results exist for inclusive jet production, dijet production as a function of dijet invariant mass and jet rapidity separation, as well as third-jet activities; see for example Ref. [5] for a recent review. In particular, new measurements have appeared on the inclusive jet cross section as a function of jet $p_{T}$ by CMS [6], and dijet production by ATLAS [7], based on the full 2011 dataset, cf. Fig. 1. Overall, the agreement of next-to-leading order (NLO) QCD predictions with data over many orders of magnitude is rather impressive. The inclusive jet cross section has been compared to predictions based on a large set of pdfs, showing in general good agreement within theoretical and experimental uncertainties. It is interesting to note that for central rapidities the experimental and theoretical uncertainties are of similar level. For this region of rapidities the CT10 set gives a rather good description of the data, while pdfs based on DIS-data, such as from the HERA1.5 and ABKM09 sets, show larger discrepancies. The 
picture is somewhat inverted at large rapidities, where the low- $x$ gluon becomes more important. In the dijet case, where the data have an impressive reach up to about $4 \mathrm{TeV}$ in dijet mass, some discrepancies are found at very large masses and large dijet rapidity separation, a region where NLO predictions probably reach their limit of applicability. A similar observation is made by a dedicated CMS analysis, which studied central jet production with the additional requirement of a second jet in the forward region [8]. They found some significant disagreements among data and MC models. Finally, ATLAS has presented a measurement of the $D^{*}$ fragmentation function [9], showing a sizable discrepancy, with MC clearly underestimating the yield in the data. This might point to a problem with the simulations for gluon splitting to charm, similarly to the observations for the b-quark case in an earlier CMS measurement of $B \bar{B}$ angular correlations [10].
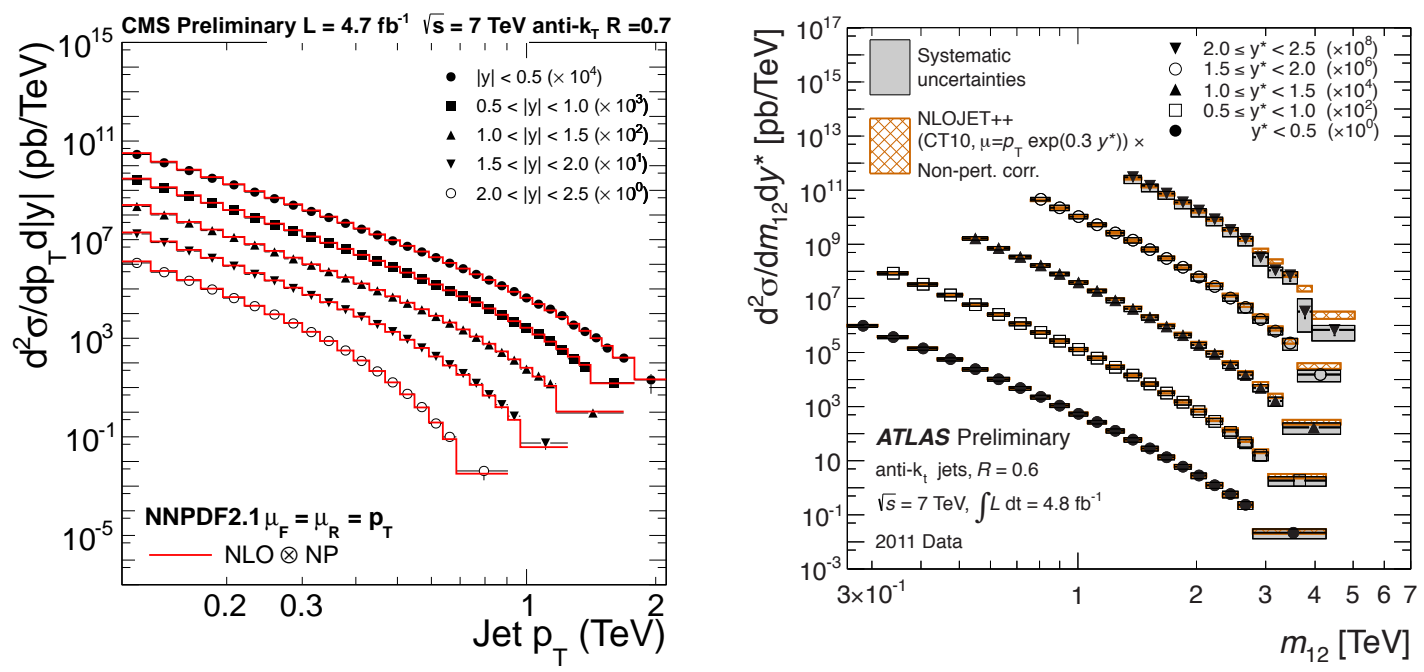

Figure 1: Left: Inclusive jet production, as a function of jet $p_{T}$ and rapidity, measured by CMS [6]; Right: ATLAS data on dijet production [7].

New results on inclusive photon, di-photon and photon plus jet production have recently been summarized in [11]. Among the highlights of this year, there is a new calculation [12] at nextto-NLO (NNLO) level for di-photon production, which finally brings the theory into agreement with data in the region of small azimuthal separation (Fig. 2, left). In that region of phase space the previously available NLO calculation is effectively a leading order approximation, which underestimates the data obtained for this distribution both at the LHC and the TEVATRON. Thus here we have a spectacular example for the need of NNLO calculations, for the description of particular variables in specific regions of phase space, not only because of radiative corrections, but also because of the appearance of new partonic channels in the initial state only at a certain order of perturbation theory. Also worth mentioning is the first LHC measurement on photon plus jet production by ATLAS [13], as a function of several kinematic variables and differential in the photon-jet angular separation. This is a classical study for hadron colliders, in particular because of the sensitivity to the gluon pdf. The data are in good agreement with NLO predictions (Fig. 2, right), besides some deviations seen for photon $p_{T}$ below $50 \mathrm{GeV}$. A similar observation had been made for inclusive photon production.

Whereas an excellent agreement of data with NNLO QCD predictions, for the inclusive pro- 

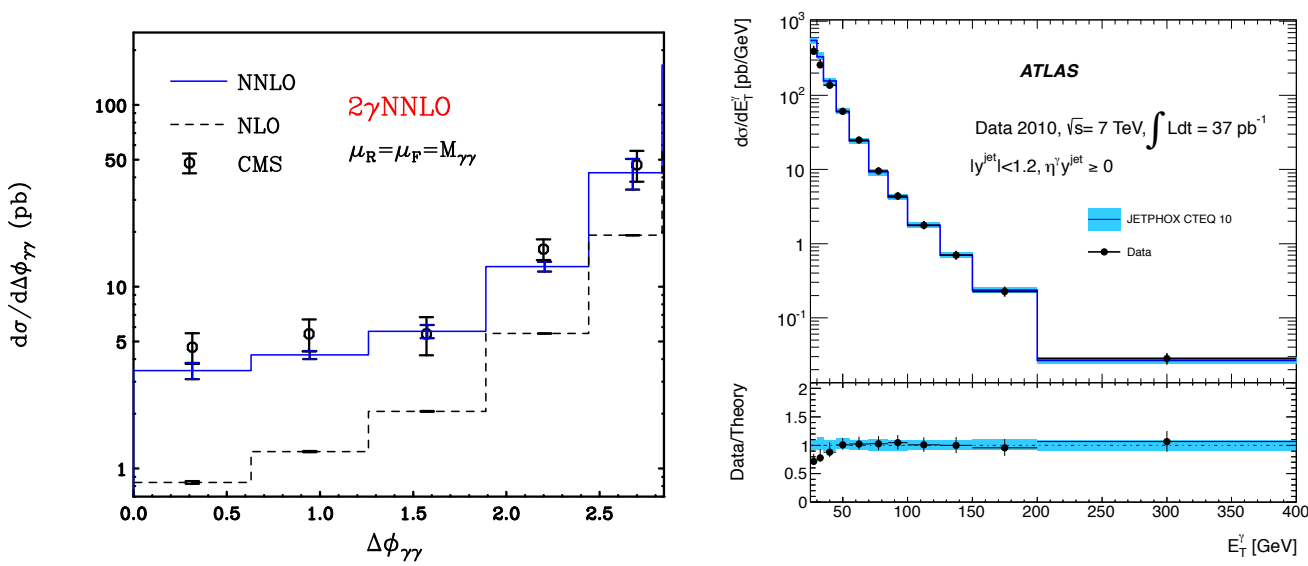

Figure 2: Left: Comparison of CMS data and QCD predictions for the di-photon azimuthal separation [12]; Right: photon plus jet production cross section from ATLAS [13].

duction of $W$ and $Z$ bosons, had already been observed in earlier studies, now more focus has been put on differential studies, eg. as a function of rapidity [14] in order to probe PDFs, as well as on the study of vector boson plus jet production [15]. These processes are extremely important backgrounds for searches of supersymmetry and the Higgs, especially for associated Higgs production in the low mass region. Furthermore, such measurements allow for testing different approaches to the implementation of perturbative QCD calculations into MC codes, such as at fixed order (NLO) or based on the matching of leading order matrix elements with parton showers, for example in MADGRAPH, ALPGEN or SHERPA. Thanks to important recent advances, NLO calculations are now available up to high jet multiplicities [16]. Concerning such jet multiplicities in $W$ (or $Z$ ) plus jet production, as well as angular correlations among the jets, overall a very good agreement with the NLO and matched calculations is found. Also dijet masses and the $H_{T}$ distribution (scalar sum of jet momenta) are well modeled over large regions of phase space, where the various calculations are applicable. For the more specific case of vector boson plus heavy flavor production $(b-$ or $c$-tagged jets), a rather consistent picture seems to appear from the TEVATRON and the LHC: data and NLO QCD predictions agree, within the sometimes sizable theoretical and experimental uncertainties, for $W+c$ and $Z+b$ production, whereas deviations are found for $W+b(b)$. This is interesting, again also because of the relevance of this process for the Higgs search.

Going lower in production cross section for electro-weak particles, the most relevant and often studied processes are di-boson production $(W \gamma, Z \gamma, \mathrm{WW}, \mathrm{WZ}, \mathrm{ZZ})$, for various decay channels of the vector bosons. The large and by now rather complete set of LHC results is summarized in more detail in Ref. [17]. The picture arising is that all the aforementioned processes, measured with statistics up to $5 \mathrm{fb}^{-1}$, are in agreement with NLO QCD predictions, which then allows to put stringent constraints on anomalous trilinear gauge couplings. There might be some hint that the measured $W W$ cross section is slightly higher (however, not at a statistically significant level) both in ATLAS and CMS, compared to the NLO predictions. Since this process is particularly relevant for the understanding of electro-weak symmetry breaking, it will be interesting to follow up on future results in this area. 


\section{Heavy Flavour Physics}

A review of heavy flavour production results from the LHC [18] reveals that, overall, perturbative QCD gives a rather satisfactory description, with still some discrepancies seen for particular phase space regions of $p_{T}$ and/or rapidity distributions. Indeed, such measurements have been carried out for inclusive open $b$ production, $B$ hadron production as well as $b$-jet production. Furthermore, angular correlations in events with two $B$-tags have shown some need for improvements in the Monte Carlo modeling of gluon splitting into $b$ quarks. Recent highlights comprise new results from CMS on $\Lambda_{b}$ production, showing a steeper $p_{T}$ spectrum than observed for $B$ mesons, the first particle discovered at the LHC, namely the $\chi_{b}(3 P)$ state, as well as new LHCb measurements [19] of $\chi_{c}, \psi(2 s)$ and double charm production. Interestingly, the latter represents a very stringent test for models of double parton scattering.

An excellent review on probing new physics with heavy flavours, and the current experimental status can be found in [20]. These efforts can be subdivided in (i) attempts to constrain the CKM parameters, (ii) measurements of direct or mixing-induced CP violation and (iii) the searches for very rare decays, such as $B_{s} \rightarrow \mu^{+} \mu^{-}, B \rightarrow K^{*} \mu^{+} \mu^{-}, B^{+} \rightarrow \pi^{+} \mu^{+} \mu^{-}$and $B \rightarrow 4 \mu$. The process $B_{s} \rightarrow \mu^{+} \mu^{-}$attracts most of the attention, since it is very sensitive to contributions from new physics such as SUSY and predicted by the SM to be $(3.2 \pm 0.2) \times 10^{-9}$. The current world's best limit, obtained by LHCb from their $1 \mathrm{fb}^{-1}$ dataset [21], is $B R\left(B_{s} \rightarrow \mu^{+} \mu^{-}\right)<4.5 \times 10^{-9}$, closely followed by CMS [22] which finds $B R\left(B_{s} \rightarrow \mu^{+} \mu^{-}\right)<7.7 \times 10^{-9}$ from their full 2011 dataset (5 $\left.\mathrm{fb}^{-1}\right)$. The strong power of this observable has, e.g., been discussed in [23, 24], showing that these recent results exclude a very large portion of parameter space for SUSY models.

In conclusion, the new results on heavy flavour physics could be summarized by naming $\mathrm{LHCb}$ as an "anomaly terminator". This is because (i) earlier indications of a large phase $\Phi_{s}$ in $B_{s}$ mixing have not been confirmed, the current results showing nice agreement with SM expectations; (ii) the measured forward-backward asymmetry and derived parameters in the $B \rightarrow K^{*} \mu^{+} \mu^{-}$decay also agree with the SM, and thus do not confirm earlier hopes of possible signs of new physics in this decay; (iii) and finally the limit on the $B_{s} \rightarrow \mu^{+} \mu^{-}$branching ratio is approaching the SM value, with a first measurement to be expected later in 2012. Nevertheless, for those believing in new physics showing up in heavy flavour systems, their is now some new hope due to the large CPasymmetry found in charm. However, care should be taken here, since SM predictions in this area suffer from large long-distance (non-perturbative) QCD effects, thus are notoriously difficult to predict, i.e. in the end it could simply turn out that the observed large asymmetry could be ascribed to such QCD effects. Overall, the phenomenologists are more and more given a fantastic set of data and experimental constraints, which allow putting strong limits on new physics, in particular when combined with other observables, such as direct searches at colliders (see below).

\section{Physics of the Top Quark}

The top quark is given special attention because of several reasons: it is by far the heaviest of all quarks, and with a mass of the order of the electro-weak scale it is conceivable that the top plays a special role in electro-weak symmetry breaking. Furthermore, it is considered to be a possibly important gateway to new physics. Until recently the TEVATRON has been the only 
player in the field. However, the LHC has quickly risen to the status of a "top factory" and the LHC experiments start to play the leading role more and more. A central test of SM predictions is the measurement of the top-pair production cross section. Lately, the LHC experiments have presented new results $[25,26]$ for a large number of channels (leptons+jets, dileptons, $\tau+\mu$, $\tau+$ jets, all hadronic), analyzing data sets between 0.7 and $4.7 \mathrm{fb}^{-1}$. The currently combined best cross section values found by ATLAS and CMS are $\sigma_{t \bar{t}}=177 \pm 3$ (stat $)_{-7}^{+8}$ (syst) \pm 7 (lumi) pb and $\sigma_{t \bar{t}}=165.8 \pm 2.2$ (stat) \pm 10.6 (syst) \pm 7.8 (lumi) pb, respectively. Here one should highlight that the experimental uncertainty has already achieved a level of $6 \%$, which is smaller than the uncertainty on the theoretical predictions.

What concerns the top mass, the TEVATRON is still leading, with the world's most precise measurement obtained from a TEVATRON combination of $m_{t}=173.2 \pm 0.6$ (stat) \pm 0.8 (syst) GeV [27], noteworthy a quark mass measurement with a relative uncertainty of $0.54 \%$. Further improvements are still expected until the final analysis of the full Run II dataset. However, the LHC is catching up. For example, as summarized in [28], CMS has come up with their latest best result of $m_{t}=172.6 \pm 0.6$ (stat) \pm 1.2 (syst) $\mathrm{GeV}$, thus already achieving the same statistical precision as the TEVATRON experiments. However, this preliminary determination does not yet consider some systematic uncertainties, such as color reconnection and underlying event effects. A somewhat "disturbing" aspect of the direct top mass determinations from kinematic reconstruction is the not really well defined meaning of the finally extracted parameter. While it is supposed to be close to a definition according to a pole-mass scheme, currently a theoretically sound understanding is not available, which triggers the question if we really know this quark mass at the $0.5 \%$ accuracy level. On the other hand, a theoretically very well defined approach is given by the extraction of the top mass (typically in the form of a running mass) from a top cross section measurement. In view of the ever improving precision on the latter, this becomes more and more interesting. So far an accuracy of $\mathscr{O}(7 \mathrm{GeV})$ is attained, mostly dominated by pdf uncertainties, and achieving a $5 \mathrm{GeV}$ error seems to be viable [29].

Besides production cross sections and mass, an amazing amount of further top properties have been studied, see e.g. [30]. These comprise spin correlations, $W$ helicity and polarization in top decays, extractions of $\left|V_{t b}\right|, m_{t}-m_{\bar{t}}$, the electric charge of the top, the charge asymmetry, searches for anomalous couplings and flavour-changing neutral currents, as well as a first study of jet veto effects in top-pair production. Basically for all these properties and observables agreement is found among data and SM predictions.

\section{Searches for New Phenomena}

The searches for new physics, now dominated by the LHC results, can be roughly classified into two large sectors, namely (i) those concentrating on signatures of SUSY particles, and (ii) the large class of searches for other particles and interactions beyond the SM. The sheer amount of SUSY exclusion plots published so far is testimony of the enormous efforts invested at the collider experiments, in order to get any hint of SUSY components in the data. Typical classifications of the analyses follow topological considerations, such as looking for events with large missing transverse energy (MET), due to the possible production of weakly interacting massive SUSY particles, accompanied by high- $p_{T}$ jets, one or two opposite or same-sign leptons, more than two 
leptons or photons. The interpretation of the, so far unsuccessful, searches of any deviation from the SM predictions, is carried out in various manners; either in the context of since long established specific SUSY incarnations, with very constrained parameter sets, such as mSUGRA or cMSSM, or in a more general approach as implemented in so-called Simplified Models (see e.g. Ref.[31]). In this case basic properties of particle cascades, arising from the decays of heavy particles such as pair-produced gluinos, are explored. In spring 2012 first results were presented based on the full 2011 statistics, showing the potential for big advances in terms of excluded parameter space. In simple terms, the current results of "generic" squark and gluino searches, in the topologies as mentioned before, allow setting limits around the $\mathrm{TeV}$ scale, if interpreted in scenarios such as the cMSSM, see e.g. [32] for a most recent example. Thus, with the first two years of LHC data this mass scale is pushed rather high, such that some start to consider giving up (at least to some extent) naturalness arguments. On the other hand, first attempts have already started, and will be pursued with much more vigor in 2012, regarding the searches for third generation squarks. So far limits in those cases are not too strong, roughly around $300 \mathrm{GeV}$. Such efforts are, e.g., motivated by models where the first generation squarks are pushed to very high mass scales, whereas only the third generation is kept light, around the electroweak scale, arguing that after all naturalness can be maintained if the effects from top loops, which dominate radiative corrections to the Higgs mass, are controlled by contributions from particles such as stops. These searches could turn out to be rather difficult, in particular if the mass separation between the top and third-generation spartners is not too large. Related to these SUSY searches, there are two further aspects worth mentioning: (i) when looking at the enormous amount of analyses, in the end always condensed into a few exclusion plots, one easily forgets to appreciate the large ingenuity and the many new ideas, which are at the basis of those results. In particular, during these last years a large set of new observables, which are differently sensitive to SM backgrounds and to the appearance of new heavy particles, have been established, as well as many clever, so-called data-driven, methods have been developed, in order to estimate SM background contributions to the search regions. In this context, also observables are studied, such as the ratio of $Z+$ jet over $\gamma+$ jet production as a function of $H_{T}$ and/or jet multiplicity, which are interesting in itself from a SM point of view.

An example of the complementarity between direct and indirect searches is discussed in [24]. This study analyzes the constraining power, in terms of SUSY models, arising from heavy flavor physics, such as rare decays $\left(B \rightarrow K^{*} \mu^{+} \mu^{-}, B_{s} \rightarrow \mu^{+} \mu^{-}\right)$mentioned above, or from searches for (supersymmetric) Higgs bosons. In simple terms, the direct searches push the masses of (first generation) particles higher and higher, and rare decays such as $B_{s} \rightarrow \mu^{+} \mu^{-}$strongly constrain $\tan \beta$ to lower values, therefore creating tension with other observables such as the muon $g-2$ result. Though, concerning the latter, there might still be need for a better understanding of the theory uncertainties, before taking this tension too seriously.

Similarly to the SUSY searches, also other attempts to look for new physics are so numerous by now that a comprehensive summary is basically impossible. Many new LHC results have been presented [33] lately, which show that exclusion limits for heavy objects, such as heavy vector bosons $\left(Z^{\prime}, W^{\prime}\right)$ or excited quarks, have reached the few-TeV range. Even higher scales are excluded in the context of certain large extra dimension models or the searches for miniature black holes. Typical exclusion limits for heavy fermions, such as 4th generation partners, are around half a TeV. For sure, the philosophy of not leaving any stone unturned, will be pursued at the new $8 \mathrm{TeV}$ LHC 
run, where the higher centre-of-mass energy leads to a significant increase of effective luminosity, in particular when searching for very heavy objects.

\section{Searches for the Higgs Boson}

A traditional approach to testing the electroweak sector of the SM is by looking at the overall consistency among direct measurements of the $W$ and top quark masses, current limits on the Higgs mass $m_{H}$, and the SM relationship among $m_{W}, m_{t}$ and $m_{H}$. The latest version of this test has been summarized in Ref. [34], showing consistency, at the 1 sigma level, among these mass measurements and a possible existence of a SM Higgs with mass around $125 \mathrm{GeV}$. The two most important new ingredients to this test are an improved measurement of $m_{W}$ at the TEVATRON and the strong Higgs exclusion limits, as discussed below. The latest, and the world's most precise, determination of the $W$ mass has been obtained by CDF [35], with an astonishing total uncertainty of $19 \mathrm{MeV}$, leading to an uncertainty on the latest TEVATRON combination (world average) of 16 $\mathrm{MeV}(15 \mathrm{MeV})$ [36].
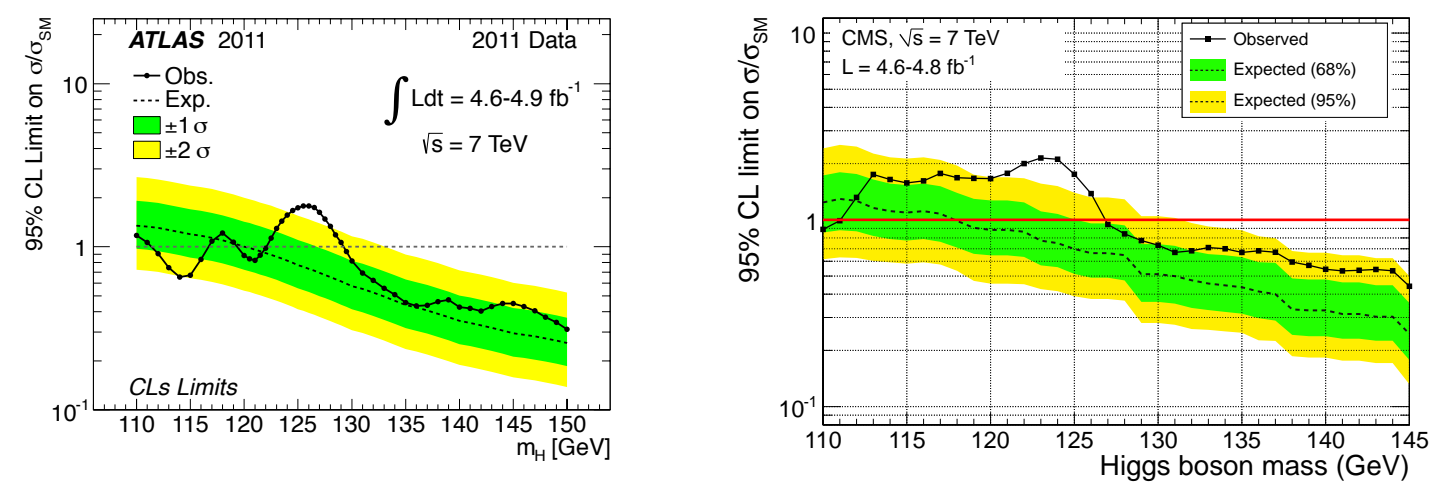

Figure 3: 95\% C.L. exclusion limits on the SM Higgs cross section, as a function of the hypothetical Higgs mass, as derived from a combination of the ATLAS [37] (left) and CMS [38] (right) Higgs searches.

At the recent winter conferences, the LHC experiments have presented the latest combinations of their Higgs searches [37, 38] : ATLAS excludes, at 95\% C.L., the mass ranges 111-117, 119-122 and $129-541 \mathrm{GeV}$ and CMS excludes the range $127-600 \mathrm{GeV}$. Very interestingly, these combined results indicate a slight excess in the mass range of roughly $122-128 \mathrm{GeV}$, with the individual significances of those excesses somewhat above the 2 sigma level (cf. Figs. 3).

It is simply impressive to see what the LHC experiments have delivered, in terms of Higgs results, over such a short time scale between the end of data taking in 2011 and the winter/spring conferences in 2012. A rather solid conclusion appears to be that a SM Higgs boson is excluded, to very high level of confidence, for masses above $\sim 130 \mathrm{GeV}$ up to about $600 \mathrm{GeV}$, where the current searches stop. As mentioned above, all experiments observe some excess in the region around 125 GeV. ${ }^{1}$

\footnotetext{
${ }^{1}$ At the time of writing these proceedings, the LHC experiments have presented updates of these searches, now including also the $8 \mathrm{TeV}$ data from 2012, and showing an exciting confirmation of those earlier excesses at the $5 \sigma$ level.
} 


\section{Conclusions}

The wealth of new data from the LHC experiments is overwhelming and exciting at the same time. So far, the Standard Model appears to be as healthy as ever, with no really significant indication for a deviation from its predictions observed, and with the final missing building block, the Higgs boson, probably on the horizon. In a year from now, our big puzzle called "particle physics up to the TeV scale" will be even more complete than already seen this year, and we might know then if there is any space left for some missing piece of the puzzle, entitled "new physics".

\section{Acknowledgments}

My sincere thanks go to the organizers of this excellent conference, for their kind invitation to give this presentation.

\section{References}

[1] G. Dissertori, arXiv:1205.2209 [hep-ex].

[2] J. M. Butterworth, G. Dissertori and G. P. Salam, arXiv:1202.0583 [hep-ex], to be published in Annu. Rev. Nucl. Part. Sci. 2012, 62.

[3] G. Aad et al. [ATLAS Collaboration], arXiv:1112.6426 [hep-ex].

[4] S. Chatrchyan et al. [CMS Collaboration], JINST 6 (2011) P11002 [arXiv:1107.4277 [physics.ins-det]].

[5] G. Jones, proceedings of the 2012 Moriond QCD conference, 2012.

[6] CMS Collab., Physics Analysis Summary CMS-PAS-QCD-11-004, 2012; http://cdsweb. cern. ch/record/1431022? ln=en.

[7] ATLAS Collab., Conference Note ATLAS-CONF-2012-021, 2012; http://cdsweb.cern.ch/record/1430730.

[8] S. Chatrchyan et al. [CMS Collaboration], JHEP 1206 (2012) 036 [arXiv:1202.0704 [hep-ex]].

[9] G. Aad et al. [ATLAS Collaboration], Phys. Rev. D 85 (2012) 052005 [arXiv:1112.4432 [hep-ex]].

[10] V. Khachatryan et al. [CMS Collaboration], JHEP 1103 (2011) 136 [arXiv:1102.3194 [hep-ex]].

[11] S. Gascon-Shotkin, proceedings of the 2012 Moriond QCD conference, 2012.

[12] S. Catani, L. Cieri, D. de Florian, G. Ferrera and M. Grazzini, Phys. Rev. Lett. 108 (2012) 072001 [arXiv:1110.2375 [hep-ph]].

[13] G. Aad et al. [ATLAS Collaboration], Phys. Rev. D 85 (2012) 092014 [arXiv:1203.3161 [hep-ex]].

[14] RAaij et al. [LHCb Collaboration], JHEP 1206 (2012) 058 [arXiv:1204.1620 [hep-ex]].

[15] A. A. Paramonov et al. [ATLAS and CMS Collaborations], arXiv:1206.1619 [hep-ex].

[16] D. Maitre, these proceedings.

[17] J. Malcles, proceedings of the 2012 Moriond QCD conference, 2012.

[18] E. Aguilo, arXiv:1205.5678 [hep-ex]. 
[19] C. Potterat, proceedings of the 2012 Moriond QCD conference, 2012.

[20] A. Schopper, proceedings of the 2012 Moriond QCD conference, 2012.

[21] R. Aaij et al. [LHCb Collaboration], Phys. Rev. Lett. 108 (2012) 231801 [arXiv:1203.4493 [hep-ex]].

[22] S. Chatrchyan et al. [CMS Collaboration], JHEP 1204 (2012) 033 [arXiv:1203.3976 [hep-ex]].

[23] D. M. Straub, arXiv:1205.6094 [hep-ph].

[24] F. Mahmoudi, arXiv:1205.3099 [hep-ph].

[25] I. Aracena, proceedings of the 2012 Moriond QCD conference, 2012.

[26] F. -P. Schilling, Int. J. Mod. Phys. A 27 (2012) 1230016 [arXiv:1206.4484 [hep-ex]].

[27] O. Brandt [CDF and D0 Collaborations], arXiv:1204.0919 [hep-ex].

[28] S. Blyweert [ATLAS and CMS Collaborations], arXiv:1205.2175 [hep-ex].

[29] M. Beneke, P. Falgari, S. Klein, J. Piclum and C. Schwinn, arXiv:1205.0988 [hep-ph].

[30] A. Lister, proceedings of the 2012 Moriond QCD conference, 2012.

[31] D. Alves et al. [LHC New Physics Working Group Collaboration], arXiv:1105.2838 [hep-ph].

[32] S. Chatrchyan et al. [CMS Collaboration], arXiv:1207.1798 [hep-ex].

[33] L. Sonnenschein, proceedings of the 2012 Moriond QCD conference, 2012.

[34] R. Lopes de Sa [CDF and D0 Collaboration], arXiv:1204.3260 [hep-ex].

[35] T. Aaltonen et al. [CDF Collaboration], Phys. Rev. Lett. 108 (2012) 151803 [arXiv:1203.0275 [hep-ex]].

[36] Tevatron Electroweak Working Group [CDF and D0 Collaborations], arXiv:1204.0042 [hep-ex].

[37] G. Aad et al. [ATLAS Collaboration], arXiv:1207.0319 [hep-ex].

[38] S. Chatrchyan et al. [CMS Collaboration], Phys. Lett. B 710 (2012) 26 [arXiv:1202.1488 [hep-ex]]. 